\title{
Model Predictive Control for biped walking
}

\author{
Pierre-Brice Wieber, INRIA
}

\section{Introduction}

One of the major difficulties in making a robot walk is keeping its balance. Not considering other important questions such as energy efficiency, keeping the balance of the robot will be the only focus of this chapter: where should the robot place its feet, how should it move its body in order to move safely in a given direction, even in case of strong perturbations?

This major difficulty comes from the contact forces with the ground, which are required for locomotion, but constrained in direction and amplitude. And as stated in [38], "The prevalence of hard constraints is accompanied by a dearth of control methods for handling them (...) Model Predictive Control is one of few suitable methods (...)". We are going to review therefore in this chapter how Model Predictive Control (MPC) is used to generate stable dynamic walking motions.

An early observation is that not all the motion of the robot is constrained. As a result, the idea of artificial synergy synthesis [59] is to assign some degrees of freedom of the robot to take care of these ground contact forces constraints, so that the rest of the motion of the robot can be realized almost independently. The original proposition was to use trunk rotations to ensure that the ground contact forces follow a pre-defined pattern, more precisely, a pre-defined trajectory of the Center of Pressure (CoP), also called the Zero Moment Point (ZMP), an approach demonstrated successfully later in Waseda University [55].

It has been argued, however, that predefining the evolution of the $\mathrm{CoP}$ is not necessary nor even desirable $[28,63]$, and trunk rotations have been shown to have a relatively weak influence on balance [13]. As a result, the prevailing option has been to handle the contact force constraints directly through the motion of the Center of Mass (CoM) of the robot. For this reason, the focus of this chapter will be on the motion of the CoM with respect to the contact points with the ground. Angular momentum will also play a role, but all the rest of the motion of the robot will generally be considered to be tackled independently with standard Inverse Kinematics or Inverse Dynamics methods.

\section{The dynamics of legged robots}

As for all robots that have the capacity to move in their environment, the configuration space of legged robots combines the configuration $\hat{q} \in \mathbb{R}^{N}$ of their $N$ joints with the position $x_{0} \in \mathbb{R}^{3}$ and orientation $\theta_{0} \in \mathbb{R}^{3}$ of a central body (pelvis or trunk) or of an extremity (foot or hand):

$$
q=\left[\begin{array}{c}
\hat{q} \\
x_{0} \\
\theta_{0}
\end{array}\right] .
$$


This structure is naturally reflected in the Lagrangian dynamics

$$
M(q)\left(\left[\begin{array}{c}
\ddot{\hat{q}} \\
\ddot{x}_{0} \\
\ddot{\theta}_{0}
\end{array}\right]+\left[\begin{array}{l}
0 \\
g \\
0
\end{array}\right]\right)+n(q, \dot{q})=\left[\begin{array}{l}
u \\
0 \\
0
\end{array}\right]+\sum_{i} C_{i}(q)^{t} f_{i}
$$

of the system, where $M(q) \in \mathbb{R}^{(N+6) \times(N+6)}$ is the generalized inertia matrix of the robot, $-g \in \mathbb{R}^{3}$ is the constant gravity acceleration vector, $n(q, \dot{q}) \in \mathbb{R}^{N+6}$ is the vector of Coriolis and centrifugal effects, $u \in \mathbb{R}^{N}$ is the vector of joint torques, and for all $i, f_{i} \in \mathbb{R}^{3}$ is a force exerted by the environment on the robot and $C_{i}(q) \in \mathbb{R}^{(N+6) \times 3}$ is the associated Jacobian matrix [62]. This specific structure has two important consequences.

The first consequence is that since the vector $u$ of joint torques has the same size as the vector $\hat{q}$ of joint positions, the whole dynamics including the global position $x_{0}$ and orientation $\theta_{0}$ appears to be underactuated if no external forces $f_{i}$ are exerted. The second consequence is that the part of this dynamics which is not directly actuated involves the Newton and Euler equations of motion of the robot taken as a whole (see [62] for detailed derivations). The Newton equation can be written in the following way:

$$
m(\ddot{c}+g)=\sum_{i} f_{i}
$$

with $m$ the total mass of the robot and $c$ the position of its Center of Mass $(\mathrm{CoM})$. The Euler equation can be expressed with respect to the CoM in the following way:

$$
\dot{L}=\sum_{i}\left(s_{i}-c\right) \times f_{i}
$$

with $s_{i}$ the points of applications of the forces $f_{i}$ and

$$
L=\sum_{k}\left(x_{k}-c\right) \times m_{k} \dot{x}_{k}+I_{k} \omega_{k}
$$

the angular momentum of the whole robot with respect to its CoM, with $\dot{x}_{k}$ and $\omega_{k}$ the translation and rotation velocities of the different parts $k$ of the robot, $m_{k}$ and $I_{k}$ their masses and inertia tensor matrices (expressed in global coordinates). The Newton equation makes it obvious that the robot needs external forces $f_{i}$ in order to move its CoM in a direction other than that of gravity. The Euler equation makes it clear that the positions of the points $s_{i}$ with respect to the CoM $c$ is important to keep the angular momentum $L$ under control.

\section{The dynamics of walking on a flat ground}

In case the forces applied by the environment on the robot are due to contacts with a flat ground, let us consider a reference frame oriented along the ground (therefore tilted if the ground is tilted), with the $z$ axis orthogonal to it. Without 
loss of generality, let us suppose that the points of contact $s_{i}$ with the ground are all such that $s_{i}^{z}=0$.

Let us consider then the sum of the Euler equation (4) and $c \times$ the Newton equation (3):

$$
m c \times(\ddot{c}+g)+\dot{L}=\sum_{i} s_{i} \times f_{i},
$$

and let us divide the result by the $z$ coordinate of the Newton equation to obtain

$$
\frac{m c \times(\ddot{c}+g)+\dot{L}}{m\left(\ddot{c}^{z}+g^{z}\right)}=\frac{\sum_{i} s_{i} \times f_{i}}{\sum_{i} f_{i}^{z}} .
$$

Since $s_{i}^{z}=0$, the $x$ and $y$ coordinates of this equation can be simplified in the following way:

$$
c^{x, y}-\frac{c^{z}}{\ddot{c}^{z}+g^{z}}\left(\ddot{c}^{x, y}+g^{x, y}\right)+\frac{1}{m\left(\ddot{c}^{z}+g^{z}\right)} R \dot{L}^{x, y}=\frac{\sum_{i} f_{i}^{z} s_{i}^{x, y}}{\sum_{i} f_{i}^{z}}
$$

with a simple rotation matrix $R=\left[\begin{array}{cc}0 & -1 \\ 1 & 0\end{array}\right]$.

On the right hand side appears the definition of the Center of Pressure (CoP) $p$ of the contact forces $f_{i}$. These contact forces are usually unilateral (the robot can push on the ground, not pull):

$$
f_{i}^{z} \geq 0
$$

what implies that the $\mathrm{CoP}$ is bound to lie in the convex hull of the contact points:

$$
p^{x, y}=\frac{\sum_{i} f_{i}^{z} s_{i}^{x, y}}{\sum_{i} f_{i}^{z}} \in \operatorname{conv}\left\{s_{i}^{x, y}\right\} .
$$

Combining this inclusion with the dynamic equation (8) reveals an Ordinary Differential Inclusion (ODI)

$$
c^{x, y}-\frac{c^{z}}{\ddot{c}^{z}+g^{z}}\left(\ddot{c}^{x, y}+g^{x, y}\right)+\frac{1}{m\left(\ddot{c}^{z}+g^{z}\right)} R \dot{L}^{x, y}=p^{x, y} \in \operatorname{conv}\left\{s_{i}^{x, y}\right\}
$$

which bounds the motion of the CoM $c$ of the robot and the variations of its angular momentum $L$ with respect to the position $s_{i}^{x, y}$ of the contact points.

\section{The dynamics of falling}

In case the robot is walking on a horizontal ground, the $z$ axis is aligned with gravity, so $g^{x, y}=0$ and the ODI (11) becomes

$$
c^{x, y}-\frac{c^{z}}{\ddot{c}^{z}+g^{z}} \ddot{c}^{x, y}+\frac{1}{m\left(\ddot{c}^{z}+g^{z}\right)} R \dot{L}^{x, y} \in \operatorname{conv}\left\{s_{i}^{x, y}\right\} .
$$


We can observe that it is linear with respect to the horizontal motion $c^{x, y}, \ddot{c}^{x, y}$ of the CoM and variations of the angular momentum $L^{x, y}$. The vertical motion $c^{z}, \ddot{c}^{z}$ of the CoM can usually be bounded, but in order to simplify the following derivations, let us suppose that the CoM moves strictly horizontally above the ground, i.e., $c^{z}$ is constant and $\ddot{c}^{z}=0$, so the ODI becomes:

$$
c^{x, y}-\frac{c^{z}}{g^{z}} \ddot{c}^{x, y}+\frac{R \dot{L}^{x, y}}{m g^{z}} \in \operatorname{conv}\left\{s_{i}^{x, y}\right\} .
$$

Variations of the angular momentum $L$ can also be bounded in the $x$ and $y$ directions, but to make things as simple as possible, let us consider these variations to be equal to 0 , so the ODI takes a very simple second order linear form:

$$
c^{x, y}-\frac{c^{z}}{g^{z}} \ddot{c}^{x, y}=p^{x, y} \in \operatorname{conv}\left\{s_{i}^{x, y}\right\} .
$$

Let us consider furthermore a situation where, for some reason (e.g. a cliff), no contact with the ground can be realized beyond a certain line (Fig. 1), with $a$ a vector orthogonal to this line and pointing beyond it. If the CoM of the robot reaches this line at a time $t_{0}$ with a speed pointing beyond it $\left(a^{t} \dot{c}^{x, y}\left(t_{0}\right)>0\right)$, this linear ODI can be integrated analytically very simply and lead to the following inequality [64], valid at all time $t \geq t_{0}$ :

$$
a^{t}\left(c^{x, y}(t)-c^{x, y}\left(t_{0}\right)\right) \geq \frac{a^{t} \dot{c}^{x, y}\left(t_{0}\right)}{\omega} \sinh \left(\omega\left(t-t_{0}\right)\right)
$$

with

$$
\omega=\sqrt{\frac{g^{z}}{c^{z}}}
$$

The right hand side is increasing exponentially with time. As a result, the position $c^{x, y}$ of the CoM is diverging exponentially in the direction of the vector $a$, leading inexorably to a fall.

\section{$5 \quad$ Viability and capturability}

The previous analysis demonstrates that the robot can be in a state where it hasn't fallen yet, but is bound to fall inexorably, so anticipation is crucial. The mathematical theory behind this key observation is the viability theory, developed in [7] for general ODIs and introduced in the analysis of walking robots in [60]: it may look obvious, but in order to avoid falling, the robot must be in a state where it can avoid falling, a so-called viable state. The problem is, anticipating which state is viable or not is generally hard if not impossible to determine.

In the simplified linear case developed above, the inequality (15) establishes that in the case of a fall, the motion of the CoM diverges exponentially, so the integral

$$
\int_{t_{0}}^{\infty}\left\|c^{(n)}(t)\right\| d t
$$




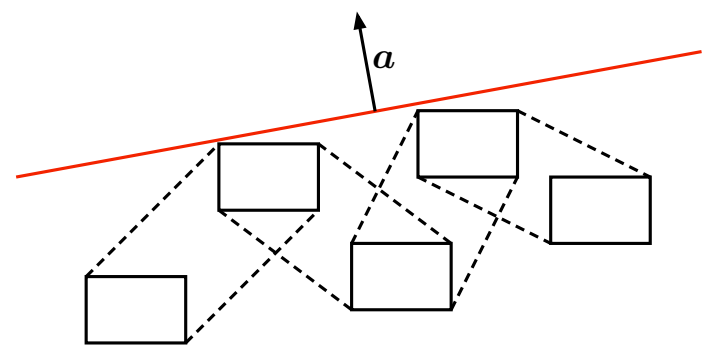

Fig. 1. A situation where, for some reason, no steps can be undertaken beyond a certain line (in red), with $a$ a vector orthogonal to this line and pointing beyond it.

of the norm of any $n^{\text {th }}$ derivative of its position would be infinite. Therefore, if we can find a finite value for this integral from a given initial state of the robot, we can conclude that this state is viable.

If we want to avoid computations over an infinite length of time, what quickly becomes unmanageable, we can focus instead on states that can be proven to be viable in a finite length of time. For example, states from which the robot is able come to a stop after a given number of steps, and therefore avoid to fall. Such states have been called capturable [48], and arguably encompass most of the states of interest for legged robots. A variant would be to consider states from which the robot is able to reach a cyclic motion, or any other state which is known to be viable in the first hand.

In the simple linear case developed above, the capturable states can be identified analytically with the help of the compound variable

$$
\xi=c+\frac{1}{\omega} \dot{c},
$$

introduced independently as the eXtrapolated Center of Mass [27], the Capture Point [47] or the divergent component of the dynamics [56]. These three denominations correspond to three key properties of this variable. First of all, a trivial reformulation gives

$$
\dot{c}=\omega(\xi-c),
$$

revealing that this point $\xi$ is the point where the CoM is converging to, hence the eXtrapolated CoM (XCoM) denomination. Following the linear dynamics (14), the horizontal motion of this point satisfies

$$
\dot{\xi}^{x, y}=\omega\left(\xi^{x, y}-z^{x, y}\right),
$$

where we can see that this point $\xi$ diverges away from the $\mathrm{CoP} z$. But if this point is above the support polygon, if we have

$$
\xi^{x, y} \in \operatorname{conv}\left\{s_{i}^{x, y}\right\},
$$


we can have $p^{x, y}=\xi^{x, y}$ so the point $\xi$ does not move, and the CoM converges to it and comes to a stop, hence the Capture Point denomination. Finally, we can observe that the second-order linear dynamics (14) can be decomposed as two first-order linear dynamics (19)-(20), the first one being stable, with the $\mathrm{CoM}$ converging to the $\mathrm{XCoM}$, the second one being unstable, with the $\mathrm{XCoM}$ diverging away from the $\mathrm{CoP}$, hence the divergent part of the dynamics denomination $[18,32]$.

The key observation here is that if the state of the robot satisfies condition (21), the robot can stop, it is capturable, viable. Further analysis can be found in [32].

\section{Model Predictive Control}

We have seen that the dynamics of walking robots is constrained by available contact forces. And as stated in [38], "Model Predictive Control is one of few suitable methods" to control such constrained systems. Considering a discretetime representation of the dynamics,

$$
x^{+}=f(x, u)
$$

where $x^{+}$is the successor state, with state and control constraints

$$
x \in \mathbb{X}, u \in \mathbb{U},
$$

Model Predictive Control usually starts with the minimization of an objective function

$$
\min _{\boldsymbol{u}} V_{f}\left(x^{\boldsymbol{u}}(N, x)+\sum_{i=0}^{N-1} l\left(x^{\boldsymbol{u}}(i, x), u(i)\right)\right.
$$

over a control sequence $\boldsymbol{u}=\{u(0), u(1), \ldots, u(N-1)\}$, where $x^{\boldsymbol{u}}(i, x)$ denotes the corresponding solution at time $i$, starting from an initial state $x$. A terminal constraint

$$
x^{u}(N, x) \in \mathcal{X}_{f}
$$

is usually considered as well. If $\boldsymbol{u}^{*}(x)$ is the optimal control sequence, the control actually applied to the system is its first element:

$$
k(x)=\boldsymbol{u}^{*}(0, x),
$$

leading to a closed loop dynamics $x^{+}=f(x, k(x))$. Important aspects of this control law are naturally stability and feasibility, i.e. satisfying constraints (23) and (25). These are easily established if a terminal cost $V_{f}(\cdot)$ and terminal constraint (25) are employed, but this is not mandatory [38,37]. In the case of walking robots, considering the viability and capturability analysis proposed earlier, it is natural to consider a terminal constraint of the form (21), and the minimization of an integral of the form (17).

Many MPC variants have been proposed, but two options are going to be significant in the following. One is based on the observation that minimizing 
an integral of the form (17) is not necessary to obtain a viable behavior: the terminal, capturability constraint can be sufficient in this respect [4]. In this case, however, it is not possible to continuously re-evaluate and adapt the motion to the state of the system: this must be triggered at distinct moments, by a carefully crafted disturbance observer, and we are going to see that this has been a limitation. The other option is to do without terminal constraint [3]: simply minimizing a truncated version of the integral (17) over a sufficiently long but finite length of time can lead to an integral decreasing with time, ensuring viability. In this case, it is possible to continuously re-evaluate and adapt the motion to the state of the system without any problem.

We are going to see in the following that the motion generation schemes that allow most of the great humanoid robots of today and yesterday to walk and run can all be seen as variants of the same MPC scheme, although they have rarely been described this way.

\section{Dynamics of the whole body, and artificial synergy synthesis}

One of the earliest approaches to computing walking and running motions was through numerical optimization [12], taking into account the dynamics of the robot, and objectives such as minimizing energy consumption. But the dynamics of the whole body of humanoid robots is so complex that the corresponding computations are too time consuming to run online. The most advanced examples, involving online optimal motion generation with the dynamics of the whole body of a Kawada HRP-2 robot, are still limited to quasi-static situations [31]. Not being able to compute walking or running motions online would limit robots to actions precomputed offline. Even with a thorough database of trajectories that could be summoned online [15], depending on the state of the robot [65], reactivity and versatility would be seriously restricted.

Alternatively, another early approach was to actually partition the problem, assigning some degrees of freedom of the robot to take care of dynamic constraints such as the ODI (11), so that the rest of the robot doesn't have to and can be operated more or less independently. This was called artificial synergy synthesis [59].

The original proposition was to use trunk rotations to ensure dynamic feasibility while the legs of the robot executed a given pre-recorded motion. More precisely, leg motions and contact points $s_{i}$ being predefined, the trajectory of the CoP $p$ could be predefined accordingly, so that it was just a matter of solving the ODE (11) for this predefined $p$ to obtain the required rotations of the trunk. This original proposition has been experimented successfully with the Waseda University WL-12R biped walking robot [55]. Predefining the trajectory of the $\mathrm{CoP}$, this method has eventually been called the ZMP approach to walking motion generation (remember the ZMP is just another name given to the CoP). This ZMP approach has been associated later with having feet always flat on 
the ground, excluding heel and toe rotation phases. Note, however, that such phases were considered in the original proposition [59].

Some aspects of this proposition can be questioned. It has been argued first of all that predefining the evolution of the CoP is not necessary nor even desirable $[28,63]$. Then, the ODI (11) clearly shows that dynamic feasibility depends on both variations of the angular momentum $L$ and motion of the CoM $c$ with respect to contact points $s_{i}$. While trunk rotations mostly involve variations of the angular momentum, a recent analysis showed that this has only a weak influence on the balance of legged robots [13]: dynamic feasibility can be handled much more efficiently by adapting the motion of the CoM, what has been the prevailing option afterwards.

The core idea of partitioning the motion of the robot builds on a profound and far-reaching observation: every part of the motion which is not involved in the dynamic constraints, everything else but angular momentum and motion of the CoM with respect to contact points, can be operated more or less independently, and appears therefore to be peripheral to the problem of legged locomotion. It is the same key observation that implicitly drives the Templates and Anchors approach and the long history of simple biomechanical models of legged locomotion that focus on a few meaningful degrees of freedom, mostly the motion of the CoM with respect to contact points, and abstract all the rest [21, $5,6]$. This idea has been tremendously successful in the legged locomotion research community, and we are going to see that all the MPC schemes described next adopt it, focusing on the motion of the $\mathrm{CoM}$ with respect to contact points, considering that the rest of the robot can be operated more or less independently.

\section{Generating walking motions on flat ground with predefined footprints and a capturability constraint}

Let us have a look first at motion generation schemes implementing the original ZMP approach, with predefined footprints, predefined CoP, and imposing a capturability constraint. Let us begin with the walking motion generation scheme implemented in the long series of Waseda University humanoid robots [55], which considers a four point mass model with predefined motion except for the horizontal motion of the waist and trunk masses which is assigned to follow a reference trajectory for the CoP. An iterative procedure based on Fast Fourier Transforms is used then to solve the dynamics

$$
\frac{\sum m_{i}\left(\ddot{c}_{i}^{z}+g^{z}\right) c_{i}^{x, y}-m_{i} c_{i}^{z} \ddot{c}_{i}^{x, y}}{\sum m_{i}\left(\ddot{c}_{i}^{z}+g^{z}\right)} \longrightarrow p_{r e f}^{x, y} .
$$

In order to execute this scheme online, a capturability constraint is introduced, imposing that the robot is always able to stop within two steps [36]. Unfortunately, details about the choice of the reference $\mathrm{CoP}$ and the exact terminal constraint are not disclosed.

The walking motion generation scheme implemented in the Munich University Johnny robot [11] considers only a three point mass model with predefined 
motion, except for the horizontal motion of the main mass in the trunk which is assigned to follow a piecewise linear reference trajectory for the CoP. One degree of freedom is left in this reference trajectory in order to impose a terminal constraint on the position of the CoM at the end of the next two steps:

$$
c^{x, y}=c_{r e f}^{x, y} .
$$

This constraint appears to be incomplete in imposing capturability, what would require to consider also the velocity of the CoM.

The walking motion generation scheme implemented in the Honda Asimo robot [56] is very similar, three point masses and a piecewise linear reference for the $\mathrm{CoP}$ with one degree of freedom left to satisfy the terminal constraint. The difference lies in the terminal constraint which is a true capturability constraint, imposing cyclicity of the motion through the Capture Point/XCoM/divergent component of the dynamics at the end of the next step:

$$
\xi^{x, y}=\xi_{\text {ref }}^{x, y} .
$$

The walking motion generation scheme implemented in the Tokyo University H7 robot [44] considers the whole dynamics (11) of the robot. The whole motion of the robot is predefined, except the horizontal motion of the CoM which is assigned to follow a reference trajectory for the CoP, set in the middle of the contact points. An iterative procedure is used then to solve the dynamics

$$
c^{x, y}-\frac{m c^{z} \ddot{c}^{x, y}-S \dot{L}^{x, y}}{m\left(\ddot{c}^{z}+g^{z}\right)} \longrightarrow \overline{s_{i}^{x, y}} .
$$

The terminal constraint (28) on the position of the CoM (incomplete with respect to capturability) is also considered at the end of the next two steps.

The walking and running motion generation scheme implemented in the Toyota Partner robot [54] is exactly the same, except for the terminal constraint which is a true capturability constraint imposing cyclicity of the motion through both the position and velocity of the CoM.

The walking and running motion generation scheme implemented in the Sony QRIO robot [41] follows a similar design, but considers only a single point mass at a constant height, with its horizontal motion assigned to minimizing the deviation of the CoP from the middle of the contact points:

$$
\min . \int\left\|c^{x, y}-\frac{c^{z}}{g^{z}} \ddot{c}^{x, y}-\overline{s_{i}^{x, y}}\right\|^{2} d t
$$

while imposing a capturability constraint on both the position and velocity of the CoM.

Another variant tested on the Kawada HRP-2 robot [39] also considers a single point mass at a constant height, but the $\mathrm{CoP}$ follows a piecewise polynomial trajectory:

$$
c^{x, y}-\frac{c^{z}}{g^{z}} \ddot{c}^{x, y}=p_{\text {ref }}^{x, y}
$$


with some degrees of freedom left to satisfy the same capturability constraint as before, through both the position and velocity of the CoM. An important characteristic of this scheme is that the piecewise polynomial trajectory of the CoP may fluctuate strongly, threatening to violate the ODI (11). An automatic adjustment of the step timings is proposed therefore in order to minimize this risk.

All of these walking and running motion generation schemes try to impose capturability through terminal constraints, but some of them appear to fail properly doing so by only constraining the position of the CoM. None of them consider an integral of the form (17): the integral (31) does not match. We have seen earlier that in this case, it is possible to re-evaluate and adapt the motion at specific instants, what has been done when having to realize new steps, or when changing the walking speed or direction. But to adapt the motion to a perturbation, a specific observer would be required to trigger the adaptation at the correct instant [4], and it appears that this option hasn't been investigated: no state feedback has been experimented with these motion generation schemes.

\section{Generating walking motions on flat ground with predefined footprints and no capturability constraint}

In the standard walking motion generation scheme implemented in the Kawada HRP-2 humanoid robot [30], the whole motion of the robot is predefined as before, except the horizontal motion of the CoM which is assigned this time to minimize the weighted integral

$$
\min . \int\left\|\dddot{c}^{x, y}\right\|^{2}+\beta\left\|c^{x, y}-\frac{c^{z}}{g^{z}} \ddot{c}^{x, y}+\frac{S \dot{L}^{x, y}}{m g^{z}}-\overline{s_{i}^{x, y}}\right\|^{2} d t
$$

of the norm of the third derivative of the motion of the CoM and the deviation of the $\mathrm{CoP}$ from a reference in the middle of the contact points. This is clearly an integral of the form (17), and we have seen that viability can be ensured in this case if we consider a truncated version of this integral over a sufficiently long but finite length of time, typically the next two steps, without the need to impose any terminal constraint. We have also seen that it is possible in this case to continuously re-evaluate and adapt the motion to the state of the system, a clear improvement over the previous approaches based solely on terminal constraints. This has been validated experimentally in various situations, effectively adapting the walking motion to perturbations [43].

Instead of computing only the motion of the CoM, this optimal control problem can be solved for its value function (the "cost-to-go"), which can be considered then as an objective function to minimize in an Inverse Dynamics control law [34]. This allows adapting the motion of the CoM to the current state of the system with relatively little online computation, and has been validated on a Boston Dynamics Atlas robot during the DARPA Robotics Challenge. 
An interesting variant [45] introduces variations of the angular momentum $L$ as an additional variable to minimize the combined integral

$$
\min . \int\left\|\dddot{c}^{x, y}\right\|^{2}+\beta\left\|c^{x, y}-\frac{c^{z}}{g^{z}} \ddot{c}^{x, y}+\frac{S \dot{L}^{x, y}}{m g^{z}}-\overline{s_{i}^{x, y}}\right\|^{2}+\gamma\left\|L^{x, y}\right\|^{2} d t,
$$

showing an improvement in the tracking of the generated motion (and the same closed loop robustness to small perturbations).

A problem however with these approaches is that simply minimizing the deviation of the $\mathrm{CoP}$ from the middle of the contact points doesn't preclude it from fluctuating, so the ODI (11) could be violated, especially in case of perturbations. This is monitored in [43] to trigger a change of footstep if necessary, but without any clear guarantee that this change is appropriate.

For this reason, it has been proposed in [63] to impose the ODI (11) as a strict constraint, considering a single point mass model with a constant height:

$$
c^{x, y}-\frac{c^{z}}{g^{z}} \ddot{c}^{x, y} \in \operatorname{conv}\left\{s_{i}^{x, y}\right\},
$$

and simply minimize the integral

$$
\min . \int\left\|\dddot{c}^{x, y}\right\|^{2} d t
$$

over a sufficiently long but finite length of time to ensure viability. This is the walking motion generation scheme implemented in the Aldebaran Nao robot [22].

A variant tested successfully on the DLR biped robot [33] considers minimizing the deviation from a reference trajectory for the $\mathrm{XCoM}$ together with the derivative of the CoP:

$$
\min . \int\left\|\xi^{x, y}-\xi_{\text {ref }}^{x, y}\right\|^{2}+\beta\left\|\dot{p}^{x, y}\right\|^{2} d t .
$$

An additional terminal constraint was considered but not tested.

In all these approaches, the footprints were predefined and kept fixed, which is obviously a strong limitation on the robot's capacity to adapt to a changing environment or to strong perturbations.

\section{Generating walking motions on flat ground with adaptive footprints}

Adapting foot placement is actually straightforward, as demonstrated on a Kawada HRP-2 robot [24]: the only required change is to consider foot placement as a decision variable, used in addition to the horizontal motion of the CoM, in order to both satisfy the ODI (35) and minimize the integral

$$
\min . \int\left\|\dot{c}^{x, y}-\dot{c}_{r e f}^{x, y}\right\|^{2} d t
$$


over a sufficiently long length of time to ensure viability (since this is clearly an integral of the form (17)), and have the CoM follow on top of that the reference velocity $\dot{c}_{r e f}^{x, y}$. Now, since the step placement is decided online, geometric feasibility needs to be checked online as well. A simple but effective option is to consider a polygonal approximation of the reachable volume of the CoM with respect to each foot on the ground [25].

Interestingly, a similar MPC scheme is used for the balance and locomotion of Aldebaran Pepper robots [35]. These humanoid robots are equipped with a single foot, resting on omnidirectional wheels. Unlike usual wheeled robots, the humanoid shape of Pepper involves a high CoM with respect to the support polygon. This poses a balance problem similar to the one of biped walking robots, asking for similar solutions: good coordination with an MPC scheme of the motion of the CoM with respect to the foot position on the ground.

Going back to biped walking robots, if desired footprints have been predefined, it is possible to use the scheme (35)-(36), and allow adaptation of foot placements when necessary, by penalizing them with a choice of norms [16, 17]. This approach was demonstrated for push recovery on a Sarcos Primus [53] and proved crucial for a Boston Dynamics Atlas robot to walk robustly over rubble and sustain significant perturbations [19].

Another approach, quite unique, starts with a singular LQR design [57], considering the classical single point mass at a constant height, assigned to minimize the deviation of the $\mathrm{CoP}$ from a combination of a reference $\mathrm{CoP}$ and the XCoM:

$$
\min . \int\left\|c^{x, y}-\frac{c^{z}}{g^{z}} \ddot{c}^{x, y}+\alpha p_{r e f}^{x, y}-(1+\alpha) \xi^{x, y}\right\|^{2} d t
$$

for some $\alpha>0$. An interesting feature of this singular LQR design is that it can be solved analytically. And an interesting property of this singular objective function is that it can be reduced to zero, in which case we have

$$
c^{x, y}-\frac{c^{z}}{g^{z}} \ddot{c}^{x, y}=(1+\alpha) \xi^{x, y}-\alpha p_{r e f}^{x, y},
$$

what can be combined with the dynamics (20) of the XCoM to obtain a stable first order dynamics

$$
\dot{\xi}^{x, y}=\alpha \omega\left(p_{\text {ref }}^{x, y}-\xi^{x, y}\right)
$$

according to which the $\mathrm{XCoM} \xi$ is going to converge to $p_{\text {ref }}^{x, y}$, and the $\mathrm{CoP} p$ as well according to (40). But in a very unusual twist, this LQR design is not used as is, and is inverted analytically to find under which condition does a piecewise constant trajectory of the $\mathrm{CoP}$ generate a non-diverging motion of the CoM. Unsurprisingly, this non-diverging condition ends up being a terminal constraint on the Capture Point/XCoM/diverging part of the dynamics, with trajectories corresponding in the end exactly to those found in [18]. But here, this terminal constraint is used in the end to decide online the step placement that will ensure viability, what is eventually validated experimentally with very strong perturbations. 
In all these schemes, foot placement and motion of the CoM are decided independently from the motion of the rest of the body. In situations involving mobile manipulation or Human Robot Interaction, it can be necessary to coordinate locomotion more closely with control objectives involving the hands or the head of the robot. But, as discussed earlier, MPC schemes taking into account the dynamics of the whole body are currently out of reach. One option then, proposed in [51], is to combine a standard Inverse Dynamics control law with the linear MPC scheme (38), in a single Lexicographic Least Squares problem. This even allows considering optional contacts, by prioritizing the contact force distribution [52]. However, this approach has been validated only in simulation.

\section{Beyond walking on a flat ground}

In all the schemes described above, the vertical motion of the CoM was predefined. Most of the times, its height was even assumed constant. This works reasonably well when walking on a flat ground, but quickly becomes an issue on non-flat ground, such as when going up and down stairs. One proposition, similar to (33), is to provide a loose reference for the 3D motion of the CoM, with predefined footprints, and minimize:

$$
\begin{aligned}
\min . \int\left\|c-c_{r e f}\right\|^{2}+\beta\left\|\dot{c}-\dot{c}_{r e f}\right\|^{2}+ & \gamma\left\|\ddot{c}-\ddot{c}_{r e f}\right\|^{2} \\
& +\delta\left\|c^{x, y}-\frac{c^{z}}{\ddot{c}^{z}+g^{z}} \ddot{c}^{x, y}-\overline{s_{i}^{x, y}}\right\|^{2} d t .
\end{aligned}
$$

This nonlinear problem is solved with a Newton-like iterative scheme (Differential Dynamic Programming), and has been validated on a Boston Dynamics Atlas robot, to walk on uneven ground during the DARPA Robotics Challenge [20]. It presents however the same challenge as the approach (33), in that simply minimizing the deviation of the CoP from the middle of the contact points doesn't preclude it from fluctuating, so the unilaterality condition (9) could be violated.

This issue has been addressed in [50], by reformulating the nonlinear ODI (11) as

$$
c^{x, y}-\zeta_{1}\left(\ddot{c}^{x, y}+g^{x, y}\right)+\zeta_{2} R \dot{L}^{x, y} \in \operatorname{conv}\left\{s_{i}^{x, y}\right\},
$$

introducing variables

$$
\zeta_{1}=\frac{c^{z}}{\ddot{c}^{z}+g^{z}}, \quad \zeta_{2}=\frac{1}{m\left(\ddot{c}^{z}+g^{z}\right)}
$$

that are constrained between iteratively adapted bounds:

$$
\underline{\zeta}_{1} \leq \zeta_{1} \leq \bar{\zeta}_{1}, \quad \underline{\zeta}_{2} \leq \zeta_{2} \leq \bar{\zeta}_{2}
$$

This allows replacing the nonlinear constraint (11) with linear constraints (43) and (45), acting separately on the horizontal and vertical parts of the motion of 
the CoM, but in a safe, conservative way. It is proposed then to simply minimize an integral

$$
\min . \int\left\|\dot{c}^{x, y}-\dot{c}_{r e f}^{x, y}\right\|^{2}+\beta\left\|c^{z}-c_{r e f}^{z}\right\|^{2} d t,
$$

with a reference horizontal velocity and a loose vertical reference for the CoM, considering a polygonal approximation of its reachable volume with respect to each foot on the ground, as in [25]. This linear MPC scheme was shown to generate human-like 3D motion of the CoM over flat ground and up and down stairs [10], but it has been validated only in simulation.

When both feet and hand supports are necessary, and the contact points $s_{i}$ are on different surfaces, we can introduce a $\mathrm{CoP}$ for each contact surface, but we can't introduce a unique $\mathrm{CoP}$ for all contact forces as we did previously. In that case, we cannot rely anymore on the ODI (11), and we need to consider instead the Newton and Euler equations (3) and (4) explicitly, together with the unilaterality condition (9), in order to check which motion is feasible or not [61, $49,26]$. This was directly applied in [23], but only to stabilize a fixed reference position of the $\mathrm{CoM}$, minimizing an integral:

$$
\min . \int\left\|c^{x, y}-c_{r e f}^{x, y}\right\|^{2}+\beta\left\|\dot{c}^{x, y}\right\|^{2}+\gamma\left\|L^{x, y}\right\|^{2}+\delta\left\|f_{i}\right\|^{2} d t,
$$

with the Newton and Euler equations linearized accordingly, and this was validated only in simulation.

As an alternative, it was observed in [40] that if the positions of the contact points $s_{i}$ are predefined, together with the vertical motion of the CoM $c^{z}$, and if we disregard the vertical component of the Euler equation (4), the horizontal Newton and Euler dynamics can be approached linearly. It was proposed then to simply minimize an integral:

$$
\min . \int\left\|c^{x, y}-c_{r e f}^{x, y}\right\|^{2}+\beta\left\|\dot{c}^{x, y}-\dot{c}_{r e f}^{x, y}\right\|^{2}+\gamma\left\|L^{x, y}-L_{r e f}^{x, y}\right\|^{2}+\delta\left\|f_{i}\right\|^{2} d t
$$

with loose references provided for the horizontal motion of the CoM and angular momentum, but with strict feasibility constraints on the contact forces. This approach has been validated on a Kawada HRP-2 robot [8], to walk in a constrained environment requiring hand supports, but as discussed earlier, having to rely on a predefined vertical motion of the CoM quickly becomes an issue on non-flat ground.

A very original approach [46] starts by observing that the products on the right side of the Euler equation (4) can be represented as differences of two convex quadratic functions (as $a^{T} b=\frac{1}{4}\|a+b\|^{2}-\frac{1}{4}\|a-b\|^{2}$ ). The Euler equation is then reformulated as

$$
\dot{L}=\sum_{i} U_{i}^{+}-U_{i}^{-}
$$

with $U_{i}^{+}$and $U_{i}^{-}$the two convex quadratic functions. It is proposed then to minimize an integral

$$
\min . \int\|\dot{c}\|^{2}+\beta\left\|U_{i}^{+}\right\|^{2}+\beta\left\|U_{i}^{-}\right\|^{2}+\gamma\left\|f_{i}\right\|^{2} d t
$$


what can be formulated as a convex Quadratically Constrained Quadratic Program (QCQP). It is proposed furthermore to borrow Mixed Integer elements from [14] to decide the contact sequence automatically. Unfortunately, the corresponding problem requires significant computation times, which have been validated only in simulations.

\section{Open questions and challenges}

We have seen that the online motion generation schemes that allow most humanoid robots to walk (and run) share a common Model Predictive Control structure, although they have rarely been described this way. All these schemes anticipate the motion of the robot for the next few steps in order to ensure viability, either through a capturability terminal constraint or through optimal control of the CoM. In all cases, the artificial synergy synthesis approach is adopted, focusing on the motion of the CoM of the robot with respect to contact points, considering that the rest of the motion can be handled more or less independently.

One of the strengths of the MPC approach is its genericity and versatility: it is easy to include additional models for force interaction with the environment or with humans [29,2], navigation aspects such as collision avoidance [42] or more advanced passive safety properties [9]. However, notwithstanding these many successes, open questions and challenges regarding the use of MPC for biped walking abound.

We have seen that using MPC beyond walking on flat ground isn't fully satisfying yet. Another important limitation in all the MPC schemes discussed here, is that the timing of the steps is always predefined, although this is known to have a huge influence on the reactivity of legged robots [32]. Some preliminary discussion has been proposed in [1], but pretty much everything still needs to be done on this aspect.

Stability and feasibility issues are almost never properly adressed, what puts most of the existing schemes at risk of sudden critical failure. Feasibility can be even more problematic for nonlinear schemes, since the satisfaction of nonlinear constraints can be particularly difficult. The approach developed in [10, 50] may be part of a solution to this problem, but this certainly needs further investigation.

Related open questions are the choice of the length of prediction, and the role of capturability, which have rarely been investigated thoroughly [66]. Regarding the first question, the current consensus is to anticipate the motion of the robot for the next two steps, but with no proof that this is the best choice in any way. Regarding the second question, we can observe that in one of the early MPC schemes [58], only kinematic and dynamic feasibility were imposed over the next two steps, and that was enough to generate stable walking and running 3D motions, with no need for capturability constraints, no need for an optimal control of the CoM, unlike all of the schemes discussed earlier. But this was tested only in simple simulations. 
In conclusion, despite the striking successes of the last decades, there is still much left to achieve and understand about Model Predictive Control of biped walking robots.

\section{Recommended Readings}

[1] Z. Aftab, T. Robert, and P.-B. Wieber. Ankle, hip and stepping strategies for humanoid balance recovery with a single Model Predictive Control scheme. In Proceedings of the IEEE-RAS International Conference on Humanoid Robots, 2012.

[2] D. J. Agravante, A. Sherikov, P.-B. Wieber, A. Cherubini, and A. Kheddar. Walking pattern generators designed for physical collaboration. In Proceedings of the IEEE International Conference on Robotics 83 Automation, 2016.

[3] M. Alamir and G. Bornard. Stability of a truncated infinite constrained receding horizon scheme: The general discrete nonlinear case. Automatica, 31(9):1353-1356, 1995.

[4] M. Alamir and N. Marchand. Numerical stabilisation of non-linear systems: Exact theory and approximate numerical implementation. European Journal of Control, 5(1):87-97, 1999.

[5] R. McN. Alexander. Mechanics of bipedal locomotion. Perspectives in Experimental Biology, 1:493-504, 1976.

[6] R. McN. Alexander. Simple models of human movement. ASME Applied Mechanics Reviews, 48(8):461-470, 1995.

[7] J.-P. Aubin. Viability Theory. Birkhäuser, 1991.

[8] H. Audren, J. Vaillant, A. Kheddar, A. Escande, K. Kaneko, and E. Yoshida. Model preview control in multi-contact motion-application to a humanoid robot. In Proceedings of the IEEE/RSJ International Conference on Intelligent Robots \&J Systems, 2014.

[9] N. Bohórquez, A. Sherikov, D. Dimitrov, and P.-B. Wieber. Safe navigation strategies for a biped robot walking in a crowd. In Proceedings of the IEEERAS International Conference on Humanoid Robots, 2016.

[10] C. Brasseur, A. Sherikov, C. Collette, D. Dimitrov, and P.-B. Wieber. A robust linear MPC approach to online generation of 3D biped walking motion. In Proceedings of the IEEE-RAS International Conference on Humanoid Robots, 2015.

[11] T. Buschmann, S. Lohmeier, M. Bachmayer, H. Ulbrich, and F. Pfeiffer. A collocation method for real-time walking pattern generation. In Proceedings of the IEEE-RAS International Conference on Humanoid Robots, 2007.

[12] C. K. Chow and D. H. Jacobson. Studies of human locomotion via optimal programming. Harvard University Technical Report No. 617, 1970.

[13] T. de Boer. Foot placement in robotic bipedal locomotion. Technische Universiteit Delft PhD thesis, 2012.

[14] R. Deits and R. Tedrake. Footstep planning on uneven terrain with mixedinteger convex optimization. In Proceedings of the IEEE-RAS International Conference on Humanoid Robots, 2014. 
[15] J. Denk and G. Schmidt. Synthesis of walking primitive databases for biped robots in 3d-environments. In Proceedings of the IEEE International Conference on Robotics \& Automation, 2003.

[16] H. Diedam, D. Dimitrov, P.-B. Wieber, K. Mombaur, and M. Diehl. Online walking gait generation with adaptive foot positioning through linear model predictive control. In Proceedings of the IEEE/RSJ International Conference on Intelligent Robots \&S Systems, 2008.

[17] D. Dimitrov, A. Paolillo, and P.-B. Wieber. Walking motion generation with online foot position adaptation based on $\ell_{1}$ - and $\ell_{\infty}$-norm penalty formulations. In Proceedings of the IEEE International Conference on Robotics 8 Automation, 2011.

[18] J. Englsberger, C. Ott, M. A. Roa, A. Albu-Schäffer, and G. Hirzinger. Bipedal walking control based on Capture Point dynamics. In Proceedings of the IEEE/RSJ International Conference on Intelligent Robots 83 Systems, 2011.

[19] S. Feng, X. Xinjilefu, C. G. Atkeson, and J. Kim. Robust dynamic walking using online foot step optimization. In Proceedings of the IEEE/RSJ International Conference on Intelligent Robots \& Systems, 2016.

[20] S. Feng, X. Xinjilefu, W. Huang, and C. G. Atkeson. 3D walking based on online optimization. In Proceedings of the IEEE-RAS International Conference on Humanoid Robots, 2013.

[21] R. J. Full and D. E. Koditschek. Templates and anchors: Neuromechanical hypotheses of legged locomotion on land. Journal of Experimental Biology, 202:3325-3332, 1999.

[22] D. Gouaillier, C. Collette, and C. Kilner. Omni-directional closed-loop walk for NAO. In Proceedings of the IEEE-RAS International Conference on Humanoid Robots, 2010.

[23] B. Henze, C. Ott, and M.A. Roa. Posture and balance control for humanoid robots in multi-contact scenarios based on model predictive control. In Proceedings of the IEEE/RSJ International Conference on Intelligent Robots $\&$ Systems, 2014.

[24] A. Herdt, H. Diedam, P.-B. Wieber, D. Dimitrov, K. Mombaur, and M. Diehl. Online walking motion generation with automatic foot step placement. Advanced Robotics, 24(5-6):719-737, 2010.

[25] A. Herdt, N. Perrin, and P.-B. Wieber. LMPC based online generation of more efficient walking motions. In Proceedings of the IEEE-RAS International Conference on Humanoid Robots, 2012.

[26] H. Hirukawa, S. Hattori, K. Harada, S. Kajita, K. Kaneko, F. Kanehiro, K. Fujiwara, and M. Morisawa. A universal stability criterion of the foot contact of legged robots - adios ZMP. In Proceedings of the IEEE International Conference on Robotics \& A Automation, pages 1976-1983, 2006.

[27] A. L. Hof, M. G. J. Gazendam, and W. E. Sinke. The condition for dynamic stability. Journal of Biomechanics, 38:1-8, 2005.

[28] Q. Huang, K. Yokoi, S. Kajita, K. Kaneko, H. Arai, N. Koyachi, and K. Tanie. Planning walking patterns for a biped robot. IEEE Transactions on Robotics and Automation, 17(3):280-289, june 2001. 
[29] A. Ibanez, P. Bidaud, and V. Padois. Unified preview control for humanoid postural stability and upper-limb interaction adaptation. In Proceedings of the IEEE/RSJ International Conference on Intelligent Robots 8 Systems, 2012.

[30] S. Kajita, F. Kanehiro, K. Kaneko, K. Fujiwara, K. Harada, K. Yokoi, and H. Hirukawa. Biped walking pattern generation by using preview control of Zero Moment Point. In Proceedings of the IEEE International Conference on Robotics \& A Automation, pages 1620-1626, september 2003.

[31] J. Koenemann, A. Del Prete, Y. Tassa, E. Todorov, O. Stasse, M. Bennewitz, and N. Mansard. Whole-body model-predictive control applied to the HRP-2 humanoid. In Proceedings of the IEEE/RSJ International Conference on Intelligent Robots $\mathcal{E}$ Systems, 2015.

[32] T. Koolen, T. de Boer, J. Rebula, A. Goswami, and J. Pratt. Capturabilitybased analysis and control of legged locomotion, part 1: Theory and application to three simple gait models. International Journal of Robotics Research, 31(9):1094-1113, 2012.

[33] M. Krause, J. Englsberger, P.-B. Wieber, and C. Ott. Stabilization of the Capture Point dynamics for bipedal walking based on model predictive control. In Proceedings of the IFAC Symposium on Robot Control, 2012.

[34] S. Kuindersma, F. Permenter, and R. Tedrake. An efficiently solvable quadratic program for stabilizing dynamic locomotion. In Proceedings of the IEEE International Conference on Robotics \&3 Automation, 2014.

[35] J. Lafaye, D. Gouaillier, and P.-B. Wieber. Linear model predictive control of the locomotion of Pepper, a humanoid robot with omnidirectional wheels. In Proceedings of the IEEE-RAS International Conference on Humanoid Robots, 2014.

[36] H. Lim, Y. Kaneshima, and A. Takanishi. Online walking pattern generation for biped humanoid robot with trunk. In Proceedings of the IEEE International Conference on Robotics \& Automation, 2002.

[37] D. Q. Mayne. Model predictive control: Recent developments and future promise. Automatica, 50:2967-2986, 2014.

[38] D. Q. Mayne, J. B. Rawlings, C. V. Rao, and P. O. M. Scokaert. Constrained model predictive control: stability and optimality. Automatica, 26(6):789$814,2000$.

[39] M. Morisawa, K. Harada, S. Kajita, K. Kaneko, F. Kanehiro, K. Fujiwara, S. Nakaoka, and H. Hirukawa. A biped pattern generation allowing immediate modification of foot placement in real-time. In Proceedings of the IEEE-RAS International Conference on Humanoid Robots, 2006.

[40] K. Nagasaka, T. Fukushima, and H. Shimomura. Whole-body control of a humanoid robot based on generalized inverse dynamics and multi-contact stabilizer that can take account of contact constraints. In Proceedings of the 17th Japanese Robotics Symposium, 2012.

[41] K. Nagasaka, Y. Kuroki, S. Suzuki, Y. Itoh, and J. Yamaguchi. Integrated motion control for walking, jumping and running on a small bipedal entertainment robot. In Proceedings of the IEEE International Conference on Robotics $\& 3$ Automation, 2004. 
[42] M. Naveau, M. Kudruss, O. Stasse, C. Kirches, K. Mombaur, and P. Souères. A reactive walking pattern generator based on nonlinear model predictive control. IEEE Robotics and Automation Letters, 2(1):10-17, 2017.

[43] K. Nishiwaki and S. Kagami. Online walking control systems for humanoids with short cycle pattern generation. International Journal of Robotics Research, 28(6):729-742, 2009.

[44] K. Nishiwaki, S. Kagami, Y. Kuniyoshi, M. Inaba, and H. Inoue. Online generation of humanoid walking motion based on a fast generation method of motion pattern that follows desired ZMP. In Proceedings of the IEEE/RSJ International Conference on Intelligent Robots \& Systems, 2002.

[45] J. Park and Y. Youm. General ZMP preview control for bipedal walking. In Proceedings of the IEEE International Conference on Robotics 8 Automation, 2007.

[46] B. Ponton, A. Herzog, S. Schaal, and L. Righetti. A convex model of humanoid momentum dynamics for multi-contact motion generation. In Proceedings of the IEEE-RAS International Conference on Humanoid Robots, 2016.

[47] J. Pratt, J. Carff, S. Drakunov, and A. Goswami. Capture point: A step toward humanoid push recovery. In Proceedings of the IEEE-RAS International Conference on Humanoid Robots, 2006.

[48] J. Pratt and R. Tedrake. Velocity based stability margins for fast bipedal walking. In Proceedings of the Ruperto Carola Symposium on Fast Motion in Biomechanics and Robotics, 2005.

[49] T. Saida, Y. Yokokoji, and T. Yoshikawa. FSW (Feasible Solution of Wrench) for multi-legged robots. In Proceedings of the IEEE International Conference on Robotics \&s Automation, 2003.

[50] D. Serra, C. Brasseur, A. Sherikov, D. Dimitrov, and P.-B. Wieber. A Newton method with always feasible iterates for nonlinear Model Predictive Control of walking in a multi-contact situation. In Proceedings of the IEEERAS International Conference on Humanoid Robots, 2016.

[51] A. Sherikov, D. Dimitrov, and P.-B. Wieber. Whole body motion controller with long-term balance constraints. In Proceedings of the IEEE-RAS International Conference on Humanoid Robots, 2014.

[52] A. Sherikov, D. Dimitrov, and P.-B. Wieber. Balancing a humanoid robot with a prioritized contact force distribution. In Proceedings of the IEEERAS International Conference on Humanoid Robots, 2015.

[53] B. J. Stephens and C. G. Atkeson. Push recovery by stepping for humanoid robots with force controlled joints. In Proceedings of the IEEE-RAS International Conference on Humanoid Robots, 2010.

[54] R. Tajima, D. Honda, and K. Suga. Fast running experiments involving a humanoid robot. In Proceedings of the IEEE International Conference on Robotics 83 Automation, 2009.

[55] A. Takanishi, M. Tochizawa, H. Karaki, and I. Kato. Dynamic biped walking stabilized with optimal trunk and waist motion. In Proceedings of the IEEE/RSJ International Conference on Intelligent Robots 83 Systems, 1989. 
[56] T. Takenaka, T. Matsumoto, and T. Yoshiike. Real time motion generation and control for biped robot -1st report: Walking gait pattern generation. In Proceedings of the IEEE/RSJ International Conference on Intelligent Robots ES Systems, 2009.

[57] J. Urata, K. Nishiwaki, Y. Nakanishi, K. Okada, S. Kagami, and M. Inaba. Online decision of foot placement using singular LQ preview regulation. In Proceedings of the IEEE-RAS International Conference on Humanoid Robots, 2011.

[58] M. van de Panne. From footprints to animation. Computer Graphics, 16(4):211-223, 1997.

[59] M. K. Vukobratović. Contribution to the study of anthropomorphic systems. Kybernetika, 8(5):404-418, 1972.

[60] P.-B. Wieber. Constrained dynamics and parametrized control in biped walking. In Proceedings of the International Symposium on Mathematical Theory of Networks and Systems, 2000.

[61] P.-B. Wieber. On the stability of walking systems. In Proceedings of the International Workshop on Humanoids and Human Friendly Robots, 2002.

[62] P.-B. Wieber. Holonomy and nonholonomy in the dynamics of articulated motion. In Proceedings of the Ruperto Carola Symposium on Fast Motion in Biomechanics and Robotics, 2005.

[63] P.-B. Wieber. Trajectory free linear model predictive control for stable walking in the presence of strong perturbations. In Proceedings of the IEEERAS International Conference on Humanoid Robots, 2006.

[64] P.-B. Wieber. Viability and predictive control for safe locomotion. In Proceedings of the IEEE/RSJ International Conference on Intelligent Robots E Systems, 2008.

[65] P.-B. Wieber and C. Chevallereau. Online adaptation of reference trajectories for the control of walking systems. Robotics and Autonomous Systems, 54(7):559-566, 2006.

[66] P. V. Zaytsev. Using Controllability of Simple Models to Generate Maximally Robust Walking-Robot Controllers. Cornell University PhD thesis, 2015. 